\title{
RIBOTS: An Automatic Telescope System for Gamma-Ray Burst Follow-Up Observations
}

\author{
Yuji Urata, Nobuyuki Kawai, Atsumasa Yoshida, Mitsuhiro Kohama \\ The Institute of Physical and Chemical Research \\ (RIKEN), Wako,Saitama 351-0198, Japan
}

Tetsuya Kawabata, Kazuya Ayani

Bisei Astronomical Observatory, Ohukura 1723-70, Bisei, Oda, Okayama 714-1411, Japan

\begin{abstract}
We are constructing a fully automatic observation system named RIBOTS (RIken-Bisei Optical Transient Seeker). We aim to detect optical flashes and early afterglows of Gamma-ray bursts (GRB) with RIBOTS. We are constructing RIBOTS with a small telescope because a quick pointing to the burst is essential for our purpose. RIBOTS is linked to the GRB alert system provided by the HETE-2 satellite.
\end{abstract}

\section{Introduction}

Gamma-ray bursts are one of the most elusive phenomena for high-energy astrophysicists. It is well known that an important clue for resolving the puzzle is the detection of transient optical emission associated with the bursts. This can be achieved by a small optical telescope system capable of rapidly moving towards the position of the event.

\section{RIBOTS}

RIBOTS is sited at Bisei Astronomical Observatory (Okayama prefecture, Japan). This system points fully automatically and starts observing after receiving a HETE-2 alert with position information. We constructed the system with a small aperture telescope and a CCD camera (Table 1). The mount slews at 6 degrees per second (max). RIBOTS will track the center of the field of view of HETE-2 $(60 \times 60 \mathrm{deg})$, so that any GRB position reported by HETE- 2 can be reached within 5 seconds after the position is available. Since HETE-2 is expected to deliver a GRB position within 10 seconds after the burst triggers, RIBOTS can start observing GRBs as early as 15 seconds from their onset.

With RIBOTS, a new optical transient will be quickly picked up in an image, and its position with 5 arcseconds accuracy will be reported automatically in 3 minutes including the time required to download the image to the computer. This refined position information will be very useful for spectroscopic observations with other, larger telescopes.

We control the system with two PCs. One PC (using Windows) controls the CCD camera while the other (using Linux) manages the whole system including weather monitoring, telescope control, time sequencing, communication with GCN and quick analysis. 
Table 1. Specifications of RIBOTS hardware

\begin{tabular}{|c|c|c|c|}
\hline \multicolumn{2}{|c|}{ Telescope Meade LX200-30 } & \multicolumn{2}{c|}{ CCD camera SBIG ST-8E } \\
\hline Aperture & $300 \mathrm{~mm}$ & Pixel & $510 \times 340(3 \times 3)$ \\
Focal length & $1000 \mathrm{~mm}$ (with 0.33 reducer) & Resolution & $5.6 \operatorname{arcsec}(3 \times 3)$ \\
FOV & $47.2^{\prime} \times 31.4$ & Read out time & $12 \sec (3 \times 3)$ \\
Slew speed & $6 \mathrm{deg} / \mathrm{sec}$ & Wavelength & $350-925 \mathrm{~nm}(\mathrm{QE} \geq 20 \%)$ \\
\hline
\end{tabular}

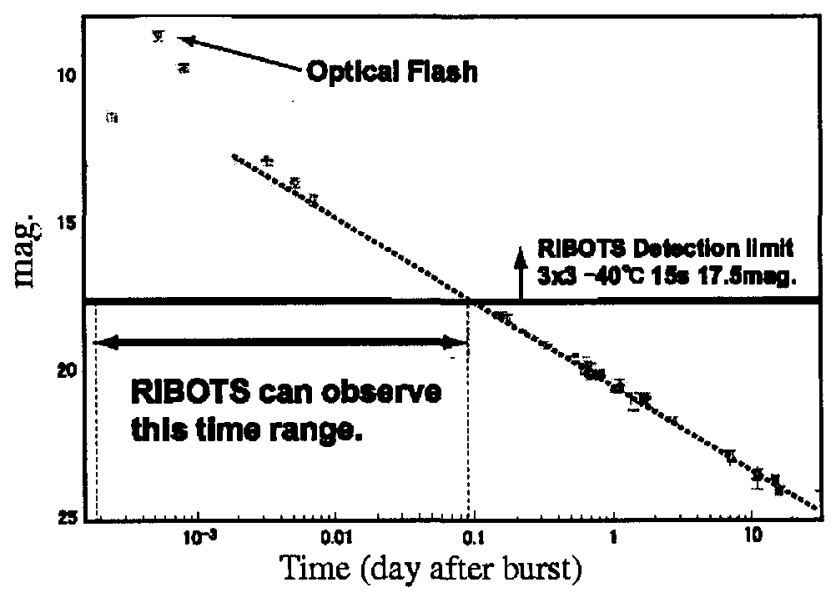

Figure 1. Comparison of the GRB990123 light curve with the RIBOTS detection limit. RIBOTS can cover the early phase of the GRB flash/afterglow (2-3 hours after the burst).

\section{Feasibility Study}

We evaluated the limiting magnitude of RIBOTS by correlating its images with the USNO-A2.0 catalog, and found it to be 17.5-18.0 mag (non-filter, 15 seconds exposure, $3 \times 3$ binning mode, dark sky at Bisei). Fig.1 shows a comparison of the GRB990123 light curve and the RIBOTS detection limit. RIBOTS has enough sensitivity to catch the optical flash and early afterglow. RIBOTS can cover the time period up to 2-3 hours after the burst, so we can obtain the light curve of the early phase afterglow phenomenon.

Considering RIBOTS's 15 sec quick pointing capability and sensitivity, RIBOTS can detect much dimmer optical flashes than that of GRB990123 (9th mag).

\section{References}

T.J.Galama, et al., Nature 398, 394 (1999)

N.Kawai, et al., this volume

T.Kawabata, et al., this volume 